\title{
3 Research Square

\section{Patients' Perceptions and Satisfaction Regarding Teleconsultations During the COVID-19 Pandemic in Jeddah, Saudi Arabia}

Mawadah M Magadmi

King Abdulaziz University Faculty of Medicine

\section{Fatemah 0 Kamel}

King Abdulaziz University Faculty of Medicine

Rania M Magadmi ( $\square$ rmagadmi@kau.edu.sa)

Pharmacology Department, Faculty of Medicine, King Abdulaziz University, P.O. Box 42751, Jeddah 21551 Saudi Arabia https://orcid.org/0000-0003-0886-4068

Research article

Keywords: COVID-19, Patient satisfaction, Teleconsultations, Saudi Arabia

Posted Date: August 27th, 2020

DOI: https://doi.org/10.21203/rs.3.rs-51755/v1

License: (c) (i) This work is licensed under a Creative Commons Attribution 4.0 International License. Read Full License 


\section{Abstract}

Background: The coronavirus disease 2019 (COVID-19) pandemic has resulted in the widespread implementation of teleconsultation. Teleconsultation provides remote communication and reduces the risk of virus transmission. However, patients' perceptions and satisfaction are relevant to ensuring the quality of the service. Thus, this study aimed to evaluate patients' perceptions and satisfaction regarding teleconsultations during the COVID-19 pandemic in Jeddah.

Methods: This was a cross-sectional study using an electronic questionnaire to assess patients' perceptions and satisfaction regarding teleconsultations during May 2020. A total of 407 participants were included.

Results: Most (38.6\%) participants were aged 40 to 59 years, women (73.2\%), and had a university education (70.8\%). Patients' perceptions of the effectiveness of teleconsultation ranged from $71.43 \%$ to $88.77 \%$ and their satisfaction level ranged between $59.40 \%$ and $83.96 \%$. Older patients who had favorable outcomes from teleconsultation strongly agreed about the effectiveness of teleconsultation and were more satisfied. Patients with higher education levels who used the service only once were more satisfied with the experience. Moreover, those patients with greater age and educational levels and those who called for emergencies and to request medication refills were more likely to use teleconsultation in the future beyond the COVID-19 pandemic. When asked about reasons for dissatisfaction, $70 \%$ of the participants were unhappy with the wait time for a teleconsultation.

Conclusions: Most patients were satisfied with the teleconsultations during the COVID-19 pandemic. However, a better understanding of patients' perceptions and reasons for dissatisfaction are fundamental in the planning and implementation of teleconsultations in Saudi Arabia.

\section{Background}

Telemedicine has been defined by the World Health Organization as "the delivery of health care services by all health care professionals using technology for the exchange of valid information for the diagnosis, treatment, and prevention of disease and injuries."[1] One of the main categories of telemedicine is teleconsultation, which is defined as interactive communication between patients and healthcare providers at a distance by using technology.[2] A recent meta-analysis has shown that teleconsultation provided a rapid alternative to face-to-face clinical visits.[3] It is considered as an effective triage method to evaluate patients' complaints, prevent unnecessary clinical visits, and reduce waiting time.[4] Although some government and private health institutes already established teleconsultation services years ago, the implementation of teleconsultation has not been popular in Saudi Arabia.[5, 6]

On March 2, 2020, Saudi Arabia announced the first case of coronavirus disease (COVID-19).[7] Shortly thereafter, the Saudi government imposed a 24-hour curfew for highly affected cities, including Jeddah. At the same time, the Ministry of Health $(\mathrm{MOH})$, as well as numerous private hospitals and clinics, have activated teleconsultation services to provide health care to citizens without leaving their homes. 
Teleconsultation is an attractive solution to minimize the risk of virus transmission,[8] and it provides not only emergency medical services but also routine medical consultations and follow-up.

The COVID-19 pandemic has offered an unexpected opportunity to increase the use of teleconsultation services in various medical specialties. Although many countries have adopted the idea of teleconsultation during the COVID-19 pandemic,[9, 10,11,12] there has been limited research in regard to patient acceptance and satisfaction. $[13,14]$ To date, no Saudi-based study has assessed patient satisfaction with this type of service during the COVID-19 pandemic. Thus, the aim of this project was to evaluate the patients' satisfaction with teleconsultation during the COVID-19 pandemic in Jeddah, Saudi Arabia. Continuing to provide teleconsultation services beyond the COVID-19 pandemic could save time and minimize the economic burden of providing health services. However, ensuring patients' satisfaction and acceptance of teleconsultation is a crucial step to ensuring success in this endeavor in Saudi Arabia. [15]

\section{Methods}

\subsection{Study design}

This cross-sectional study was conducted in Jeddah in May 2020. An electronic questionnaire was distributed online through social media (WhatsApp and Twitter). All the participants included in the study were 18 years or older, lived in Jeddah, and used teleconsultation services at least once. At the beginning of the questionnaire, the participants were briefly informed of the aim of the study and the term "teleconsultation" was clearly defined as the telephonic communication between patient and healthcare providers. Further, participants were asked to complete the questionnaire if they agreed to share their answers. Any incomplete questionnaires were excluded. This study was approved by the Unit of Biomedical Ethics Research Committee at the Faculty of Medicine, King Abdulaziz University (Reference No. 229 - 20), dated May 4, 2020.

\subsection{Sample size}

The sample size was calculated based on $\mathrm{MOH}$ reports regarding the (937) hotline center that showed the number of medical calls in Jeddah was 906 per week.[7] The estimated sample size was 347, based on achieving a confidence level ( $P$-value) of $5 \%$ and a confidence interval of $95 \%$.

\subsection{Questionnaire}

For this study, a validated electronic questionnaire was used. The validity and internal consistency of the questionnaire were tested during a pilot study on 30 subjects who were excluded from the main study. The pretesting of the questionnaire showed that the Cronbach's alpha scores for all items were above 0.7. The questionnaire was translated from English into Arabic using the backward-forward method and was double-checked by the authors and a translator. 
The questionnaire consisted of 18 questions distributed in 4 domains. The first question was a screening question to ensure the inclusion criteria (adult, living in Jeddah, and used teleconsultation service during COVID-19 pandemic). The first domain comprised demographic characteristics, including age, sex, nationality, and education level. The second domain was related to the personal experience of the teleconsultation (the number of times teleconsultation services were used during the COVID-19 pandemic, the reason for the call, and the outcome). The third and fourth domains addressed patients' perspectives regarding the effectiveness of teleconsultation and their satisfaction regarding the teleconsultation. Respondents were asked to answer from 'strongly agree' to 'strongly disagree' on a 5-point Likert scale. The maximum total score was 25 and the minimum total score was 5 , with higher scores reflecting greater satisfaction. Based on the results of this questionnaire, the participants were classified as follows: satisfied (satisfaction score from 25 to 16), neutral (satisfaction score from 15 to 11), and unsatisfied (satisfaction score from 10 to 5). Moreover, the Likert scores were transformed into satisfaction percentages using the following formula: Satisfaction $\%=($ strongly agree + agree $) /$ all response $\times 100$.

\subsection{Statistical analysis}

Data cleaning and data analysis for this study were performed using the Statistical Package for Social Sciences (SPSS) v. 23.0. Descriptive statistics were used to characterize the study population. Demographic data were described with frequency and percentages, while satisfaction scores were described using mean (M) and standard deviation (SD). Comparisons between the two groups were represented by the Mann-Whitney $U$ test. A $P$-value $<.05$ was considered statistically significant. Qualitative thematic analysis for open-ended questions was used.

\section{Results}

A total of 407 responses (339 in Arabic and 68 in English) were received during the study period. Eleven questionnaires were excluded from the analysis due to incomplete information. The completion rate was $97.3 \%$

\subsection{Demographic characteristics}

The results obtained for the demographic data are listed in Table 1. The participants' age range was 18 to older than 60 years. Most (38.6\%) of the participants were aged 40 to 59 years, 28.3\% were 18 to 29, and $25.9 \%$ were 30 to 39 . Some $73.2 \%$ were women. Most (92.5\%) participants were Saudi nationals. Most (70.8\%) participants had a university education.

The prevalence of using teleconsultation during the COVID-19 pandemic showed that almost $70 \%$ used the service one time only, whereas $30 \%$ used this service more than once. The main reason for using the service was experiencing a new complaint (26\%), next, for follow-up $(24 \%)$, then for emergencies and for COVID-19-related medical issues at $18 \%$ each, and finally $14 \%$ for medication refills. 
The most common outcome from the teleconsultation was prescribed drugs (39.2\%), followed by reassurance and self-management (35.2\%). Making an appointment (5.1\%) and sending for an ambulance (3.6\%) were the least common reasons for using the service. 
Table 1

Demographic characteristics.

\begin{tabular}{|c|c|c|c|}
\hline Characteristics & & $\begin{array}{l}\text { Frequency } \\
(\mathrm{N})\end{array}$ & $\begin{array}{l}\text { Percentage } \\
(\%)\end{array}$ \\
\hline \multirow[t]{4}{*}{ Age } & $18-29$ & 94 & $28.3 \%$ \\
\hline & $30-39$ & 86 & $25.9 \%$ \\
\hline & $40-59$ & 128 & $38.6 \%$ \\
\hline & $>60$ & 24 & $7.2 \%$ \\
\hline \multirow[t]{2}{*}{ Sex } & Men & 89 & $26.8 \%$ \\
\hline & Women & 243 & $73.2 \%$ \\
\hline \multirow[t]{2}{*}{ Nationality } & Saudi & 307 & $92.5 \%$ \\
\hline & Non-Saudi & 25 & $7.5 \%$ \\
\hline \multirow[t]{4}{*}{ Educational level } & Elementary & 2 & $0.6 \%$ \\
\hline & Secondary & 37 & $11.1 \%$ \\
\hline & University & 235 & $70.8 \%$ \\
\hline & Higher education & 58 & $17.5 \%$ \\
\hline \multirow[t]{2}{*}{ How many times did you use the service? } & Once & 232 & $69.9 \%$ \\
\hline & More than one & 100 & $30.1 \%$ \\
\hline \multirow[t]{5}{*}{ For what reason did you use the service? } & New complaint & 86 & $25.9 \%$ \\
\hline & Follow-up & 80 & $24.1 \%$ \\
\hline & Emergency & 60 & $18.1 \%$ \\
\hline & Refill medication & 46 & $13.9 \%$ \\
\hline & COVID-19 related & 60 & $18.1 \%$ \\
\hline \multirow[t]{5}{*}{$\begin{array}{l}\text { What was the outcome or treatment from } \\
\text { your call consultation? }\end{array}$} & $\begin{array}{l}\text { Reassurance and self- } \\
\text { management }\end{array}$ & 117 & $35.2 \%$ \\
\hline & Prescribed drugs & 130 & $39.2 \%$ \\
\hline & Appointment & 17 & $5.1 \%$ \\
\hline & Ambulance sent & 12 & $3.6 \%$ \\
\hline & Other & 56 & $16.9 \%$ \\
\hline
\end{tabular}

\subsection{Participants' satisfaction scores for various components of the teleconsultation}


Table 2 shows the participants' perceptions of the effectiveness of the telemedicine offered. They stated they mostly agreed to strongly agreed regarding their ability to explain their medical problems to the doctor on the phone, with a median score of 4.33 out of 5 . In addition, they believed that doctors understood their problems via telephone and were confident that the doctor could provide an appropriate management plan via teleconsultation, with median scores of 4.22 and 4.04 , respectively. They expressed satisfaction with how much the doctors told them about the treatment, with a median score of 4.03 . The lowest levels of agreement related to understanding their illness after the teleconsultation, with a median score of 3.91. Overall satisfaction rates with the service exceeded $70 \%$.

Table 2

Satisfaction scores on perceptions of the effectiveness of telemedicine $(\mathrm{N}=332)$.

\begin{tabular}{|l|ccl|}
\hline Components & Mean & SD & $\begin{array}{l}\text { Satisfaction } \\
\%\end{array}$ \\
\hline I easily explained my medical problem to the doctor on the phone. & 4.33 & 0.76 & 88.47 \\
\hline $\begin{array}{l}\text { I believe the doctor understood my medical problem on the phone. } \\
\begin{array}{l}\text { I was confident that the doctor could provide an appropriate } \\
\text { management plan via phone consultation. }\end{array}\end{array}$ & 4.22 & 0.84 & 83.71 \\
\hline $\begin{array}{l}\text { The doctor told me everything about my treatment. } \\
\text { I understood my illness much better after the phone consultation. }\end{array}$ & 4.02 & 75.19 \\
\hline Overall Medical Satisfaction & 4.03 & 0.95 & 74.44 \\
\hline
\end{tabular}

Table 3 shows the participants' satisfaction with the telemedicine service. They were the least satisfied with sharing private or personal information, with a median score of 3.7 out of 5 . However, there was greater satisfaction regarding the time offered for the consultation and the $24 / 7$ availability of consultations, with median scores of 3.99 and 4.04 out of 5, respectively. The participants were satisfied that good medical service was provided that not require transportation, and that they would likely use it again in the future after the COVID-19 pandemic, with median scores of 4.3 and 4.05 , respectively. Moreover, satisfaction rates exceeded $70 \%$ for all aspects assessed, except for the item related to sharing private information with a doctor face-to-face versus via the telephone. Some $59 \%$ of participants felt that they were more comfortable sharing private data via the phone than in face-to-face meetings. 
Table 3

Participants' satisfaction with the telemedicine service $(\mathrm{N}=332)$.

\begin{tabular}{|c|c|c|c|}
\hline Components & Mean & SD & $\begin{array}{l}\text { Satisfaction } \\
\%\end{array}$ \\
\hline $\begin{array}{l}\text { I was more comfortable telling the doctor about private or personal } \\
\text { information than in a face-face appointment }\end{array}$ & 3.70 & 1.13 & 59.40 \\
\hline I was satisfied with the consultation time. & 3.99 & 0.98 & 77.94 \\
\hline Medical consultations were offered at my preferred time 24/7. & 4.04 & 1.02 & 77.19 \\
\hline $\begin{array}{l}\text { Teleconsultation provided good medical service that did not require } \\
\text { transportation. }\end{array}$ & 4.30 & 0.88 & 83.96 \\
\hline $\begin{array}{l}\text { I would like to use teleconsultation again in the future after the } \\
\text { COVID-19 pandemic. }\end{array}$ & 4.05 & 1.12 & 76.19 \\
\hline Overall satisfaction & 4.02 & 0.8 & \\
\hline
\end{tabular}

Table 4 shows the factors affecting participants' perception of the effectiveness of teleconsultation. A significant association was observed between satisfaction levels and age $(P=.037)$, whereby older people were more likely to report higher levels of satisfaction. 
Table 4

Factors affecting participants' perception toward telemedicine satisfaction.

\begin{tabular}{|c|c|c|c|c|c|}
\hline Characteristics & & $\begin{array}{l}\text { Satisfied } \\
(25-16)\end{array}$ & $\begin{array}{l}\text { Neutral } \\
(15- \\
11)\end{array}$ & $\begin{array}{l}\text { Unsatisfied } \\
(10-5)\end{array}$ & $\begin{array}{l}P \text {. } \\
\text { value }\end{array}$ \\
\hline \multirow[t]{4}{*}{ Age } & $18-29$ & $\begin{array}{l}75 \\
(79.8 \%)\end{array}$ & $\begin{array}{l}16 \\
(17 \%)\end{array}$ & $3(3.2 \%)$ & \multirow[t]{4}{*}{.037} \\
\hline & $30-39$ & $\begin{array}{l}80 \\
(93 \%)\end{array}$ & $6(7 \%)$ & $0(0 \%)$ & \\
\hline & $40-59$ & $\begin{array}{l}114 \\
(89.1 \%)\end{array}$ & $\begin{array}{l}8 \\
(6.3 \%)\end{array}$ & $6(4.7 \%)$ & \\
\hline & $>60$ & $\begin{array}{l}22 \\
(91.7 \%)\end{array}$ & $\begin{array}{l}2 \\
(8.3 \%)\end{array}$ & $0(0 \%)$ & \\
\hline \multirow[t]{2}{*}{ Sex } & Men & $\begin{array}{l}75 \\
(84.3 \%)\end{array}$ & $\begin{array}{l}13 \\
(14.6 \%)\end{array}$ & $1(1.1 \%)$ & \multirow[t]{2}{*}{.111} \\
\hline & Women & $\begin{array}{l}216 \\
(88.9 \%)\end{array}$ & $\begin{array}{l}19 \\
(7.8 \%)\end{array}$ & $8(3.3 \%)$ & \\
\hline \multirow[t]{2}{*}{ Nationality } & Saudi & $\begin{array}{l}270 \\
(87.9 \%)\end{array}$ & $\begin{array}{l}28 \\
(9.1 \%)\end{array}$ & $9(2.9 \%)$ & \multirow[t]{2}{*}{.385} \\
\hline & Non-Saudi & $21(84 \%)$ & $4(16 \%)$ & $0(0 \%)$ & \\
\hline \multirow[t]{4}{*}{ Educational level } & Elementary & $\begin{array}{l}2 \\
(100 \%)\end{array}$ & $0(0 \%)$ & $0(0 \%)$ & \multirow[t]{4}{*}{.606} \\
\hline & Secondary & $\begin{array}{l}33 \\
(89.2 \%)\end{array}$ & $\begin{array}{l}3 \\
(8.1 \%)\end{array}$ & $1(2.7 \%)$ & \\
\hline & University & $\begin{array}{l}201 \\
(85.5 \%)\end{array}$ & $\begin{array}{l}26 \\
(11.1 \%)\end{array}$ & $8(3.4 \%)$ & \\
\hline & Higher education & $\begin{array}{l}55 \\
(94.8 \%)\end{array}$ & $\begin{array}{l}3 \\
(5.2 \%)\end{array}$ & $0(0.0 \%)$ & \\
\hline \multirow[t]{2}{*}{$\begin{array}{l}\text { How many times did you use the } \\
\text { service? }\end{array}$} & Once & $\begin{array}{l}202 \\
(87.1 \%)\end{array}$ & $\begin{array}{l}25 \\
(10.8 \%)\end{array}$ & $5(2.2 \%)$ & \multirow[t]{2}{*}{.379} \\
\hline & More than once & $\begin{array}{l}89 \\
(89 \%)\end{array}$ & $7(7 \%)$ & $4(4 \%)$ & \\
\hline \multirow[t]{3}{*}{$\begin{array}{l}\text { For what reason did you use the } \\
\text { service? }\end{array}$} & New complaint & $\begin{array}{l}75 \\
(87.2 \%)\end{array}$ & $\begin{array}{l}10 \\
(11.6 \%)\end{array}$ & $1(1.2 \%)$ & \multirow[t]{3}{*}{.145} \\
\hline & Follow-up & $\begin{array}{l}71 \\
(88.8 \%)\end{array}$ & $4(5 \%)$ & $5(6.3 \%)$ & \\
\hline & Emergency & $\begin{array}{l}52 \\
(86.7 \%)\end{array}$ & $\begin{array}{l}7 \\
(11.7 \%)\end{array}$ & $1(1.7 \%)$ & \\
\hline
\end{tabular}




\begin{tabular}{|c|c|c|c|c|c|}
\hline \multicolumn{2}{|l|}{ Characteristics } & \multirow{2}{*}{ 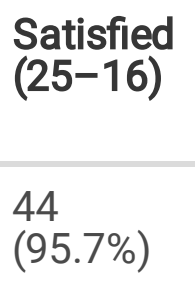 } & \multirow{2}{*}{$\begin{array}{l}\text { Neutral } \\
(15- \\
11) \\
2 \\
(4.3 \%)\end{array}$} & \multirow{2}{*}{ 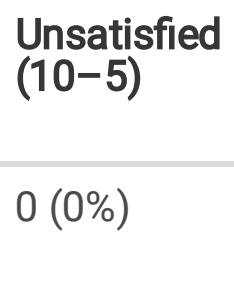 } & \multirow[t]{3}{*}{$\begin{array}{l}P \\
\text { value }\end{array}$} \\
\hline & Refill medication & & & & \\
\hline & COVID-19 related & $\begin{array}{l}49 \\
(81.7 \%)\end{array}$ & $9(15 \%)$ & $2(3.3 \%)$ & \\
\hline \multirow{5}{*}{$\begin{array}{l}\text { What was the outcome or } \\
\text { treatment from your call } \\
\text { consultation? }\end{array}$} & $\begin{array}{l}\text { Reassurance and } \\
\text { self-management }\end{array}$ & $\begin{array}{l}107 \\
(91.5 \%)\end{array}$ & $\begin{array}{l}9 \\
(7.7 \%)\end{array}$ & $1(0.9 \%)$ & \multirow[t]{5}{*}{.000} \\
\hline & Prescribed drugs & $\begin{array}{l}124 \\
(95.4 \%)\end{array}$ & $\begin{array}{l}6 \\
(4.6 \%)\end{array}$ & $0(0 \%)$ & \\
\hline & Appointment & $\begin{array}{l}15 \\
(88.2 \%)\end{array}$ & $\begin{array}{l}1 \\
(5.9 \%)\end{array}$ & $1(5.9 \%)$ & \\
\hline & Ambulance sent & $\begin{array}{l}12 \\
(100 \%)\end{array}$ & $0(0 \%)$ & $0(0 \%)$ & \\
\hline & Other & $\begin{array}{l}33 \\
(58.9 \%)\end{array}$ & $\begin{array}{l}16 \\
(28.6 \%)\end{array}$ & $7(12.5 \%)$ & \\
\hline
\end{tabular}

Furthermore, we observed a significant relationship between levels of satisfaction and outcomes from teleconsultation $(P=.000)$, in which the highest satisfaction was shown in cases with consultation for an ambulance, then for prescribed drugs, and then for reassurance and self-management. There was no significant relationship between satisfaction and any other variable.

\subsection{Factors affecting participants' satisfaction with the telemedicine experience}

Table 5 shows 3 factors significantly affecting participant satisfaction with the telemedicine services. Participants with higher educational levels were more likely to be satisfied with the service compared with those with a lower educational background $(P=.001)$. On the other hand, participants who used the service more than once were more likely to report lower satisfaction compared with those who used the service one time only $(P=.02)$. There was also a significant relationship between the level of satisfaction and the outcome of the call consultation $(P=.000)$, in which satisfaction was highest among cases with reassurance and self-management and prescribed drugs as the outcomes. 
Table 5

Factors affecting participants' satisfaction regarding telemedicine experience.

\begin{tabular}{|c|c|c|c|c|c|}
\hline Characteristics & & $\begin{array}{l}\text { Satisfied } \\
(25-16)\end{array}$ & $\begin{array}{l}\text { Neutral } \\
\text { (15- } \\
11)\end{array}$ & $\begin{array}{l}\text { Unsatisfied } \\
(10-5)\end{array}$ & $\begin{array}{l}P \text { - } \\
\text { value }\end{array}$ \\
\hline \multirow[t]{4}{*}{ Age } & $18-29$ & $\begin{array}{l}76 \\
(80.9 \%)\end{array}$ & $\begin{array}{l}15 \\
(16 \%)\end{array}$ & $3(3.2 \%)$ & \multirow[t]{4}{*}{.092} \\
\hline & $30-39$ & $\begin{array}{l}79 \\
(91.9 \%)\end{array}$ & $6(7 \%)$ & $1(1.2 \%)$ & \\
\hline & $40-59$ & $\begin{array}{l}115 \\
(89.8 \%)\end{array}$ & $\begin{array}{l}7 \\
(5.5 \%)\end{array}$ & $6(4.7 \%)$ & \\
\hline & $>60$ & $\begin{array}{l}22 \\
(91.7 \%)\end{array}$ & $\begin{array}{l}2 \\
(8.3 \%)\end{array}$ & $0(0 \%)$ & \\
\hline \multirow[t]{2}{*}{ Sex } & Men & $\begin{array}{l}79 \\
(88.8 \%)\end{array}$ & $8(9 \%)$ & $2(2.2 \%)$ & \multirow[t]{2}{*}{.884} \\
\hline & Women & $\begin{array}{l}213 \\
(87.7 \%)\end{array}$ & $\begin{array}{l}22 \\
(9.1 \%)\end{array}$ & 8 (3.3\%) & \\
\hline \multirow[t]{2}{*}{ Nationality } & Saudi & $\begin{array}{l}268 \\
(87.3 \%)\end{array}$ & $\begin{array}{l}29 \\
(9.4 \%)\end{array}$ & $10(3.3 \%)$ & \multirow[t]{2}{*}{.412} \\
\hline & Non-Saudi & $\begin{array}{l}24 \\
(96 \%)\end{array}$ & $1(4 \%)$ & $0(0 \%)$ & \\
\hline \multirow[t]{4}{*}{ Educational level } & Elementary & $0(0 \%)$ & $\begin{array}{l}2 \\
(100 \%)\end{array}$ & $0(0 \%)$ & \multirow[t]{4}{*}{.001} \\
\hline & Secondary & $\begin{array}{l}32 \\
(86.5 \%)\end{array}$ & $\begin{array}{l}3 \\
(8.1 \%)\end{array}$ & $2(5.4 \%)$ & \\
\hline & University & $\begin{array}{l}205 \\
(87.2 \%)\end{array}$ & $\begin{array}{l}22 \\
(9.4 \%)\end{array}$ & $8(3.4 \%)$ & \\
\hline & Higher education & $\begin{array}{l}55 \\
(94.8 \%)\end{array}$ & $\begin{array}{l}3 \\
(5.2 \%)\end{array}$ & $0(0 \%)$ & \\
\hline \multirow[t]{2}{*}{$\begin{array}{l}\text { How many times did you use the } \\
\text { service? }\end{array}$} & Once & $\begin{array}{l}208 \\
(89.7 \%)\end{array}$ & $\begin{array}{l}21 \\
(9.1 \%)\end{array}$ & $3(1.3 \%)$ & \multirow[t]{2}{*}{.020} \\
\hline & More than once & $\begin{array}{l}84 \\
(84 \%)\end{array}$ & $9(9 \%)$ & $7(7 \%)$ & \\
\hline \multirow[t]{3}{*}{$\begin{array}{l}\text { For what reason did you use the } \\
\text { service? }\end{array}$} & New complaint & $\begin{array}{l}76 \\
(88.4 \%)\end{array}$ & $\begin{array}{l}9 \\
(10.5 \%)\end{array}$ & $1(1.2 \%)$ & \multirow[t]{3}{*}{.519} \\
\hline & Follow-up & $\begin{array}{l}67 \\
(83.8 \%)\end{array}$ & $8(10 \%)$ & $5(6.3 \%)$ & \\
\hline & Emergency & $\begin{array}{l}53 \\
(88.3 \%)\end{array}$ & $\begin{array}{l}5 \\
(8.3 \%)\end{array}$ & $2(3.3 \%)$ & \\
\hline
\end{tabular}




\begin{tabular}{|c|c|c|c|c|c|}
\hline \multicolumn{2}{|l|}{ Characteristics } & \multirow{2}{*}{$\begin{array}{l}\text { Satisfied } \\
(25-16)\end{array}$} & \multirow{2}{*}{$\begin{array}{l}\text { Neutral } \\
(15- \\
11) \\
2 \\
(4.3 \%)\end{array}$} & \multirow{2}{*}{$\begin{array}{l}\text { Unsatisfied } \\
(10-5) \\
0(0 \%)\end{array}$} & \multirow[t]{3}{*}{$\begin{array}{l}P \\
\text { value }\end{array}$} \\
\hline & Refill medication & & & & \\
\hline & COVID-19 related & $\begin{array}{l}52 \\
(86.7 \%)\end{array}$ & $6(10 \%)$ & $2(3.3 \%)$ & \\
\hline \multirow{5}{*}{$\begin{array}{l}\text { What was the outcome or } \\
\text { treatment from your call } \\
\text { consultation? }\end{array}$} & $\begin{array}{l}\text { Reassurance and } \\
\text { self-management }\end{array}$ & $\begin{array}{l}108 \\
(92.3 \%)\end{array}$ & $\begin{array}{l}8 \\
(6.8 \%)\end{array}$ & $1(0.9 \%)$ & \multirow[t]{5}{*}{.000} \\
\hline & Prescribed drugs & $\begin{array}{l}121 \\
(93.1 \%)\end{array}$ & $\begin{array}{l}8 \\
(6.2 \%)\end{array}$ & $1(0.8 \%)$ & \\
\hline & Appointment & $\begin{array}{l}15 \\
(88.2 \%)\end{array}$ & $\begin{array}{l}2 \\
(11.8 \%)\end{array}$ & $0(0 \%)$ & \\
\hline & Ambulance sent & $\begin{array}{l}10 \\
(83.3 \%)\end{array}$ & $\begin{array}{l}2 \\
(16.7 \%)\end{array}$ & $0(0 \%)$ & \\
\hline & Other & $\begin{array}{l}38 \\
(67.9 \%)\end{array}$ & $\begin{array}{l}10 \\
(17.9 \%)\end{array}$ & $8(14.3 \%)$ & \\
\hline
\end{tabular}

3.5 Characteristics of those who would be likely to use teleconsultation again in the future after the COVID-19 pandemic

Table 6 shows the characteristics of those who indicated they would likely use the phone consultation services again in the future after the COVID-19 pandemic. A total of 5 factors were shown to have an effect on this behavior: age, educational level, times of use of phone consultation, reasons for using the phone consultation, and outcomes from the phone consultation $(P<.05)$. 
Table 6

Characteristics of those likely to use teleconsultation again in the future after the COVID-19 pandemic.

\begin{tabular}{|c|c|c|c|c|c|}
\hline Characteristics & & $\begin{array}{l}\text { Strongly } \\
\text { agree/agree }\end{array}$ & $\begin{array}{l}\text { Not } \\
\text { sure }\end{array}$ & $\begin{array}{l}\text { Strongly } \\
\text { disagree/disagree }\end{array}$ & $\begin{array}{l}P \text {. } \\
\text { value }\end{array}$ \\
\hline \multirow[t]{4}{*}{ Age } & $18-29$ & $65(69.1 \%)$ & $\begin{array}{l}11 \\
(11.7 \%)\end{array}$ & $18(19.1 \%)$ & \multirow[t]{4}{*}{.044} \\
\hline & $30-39$ & 68 (79.1\%) & $\begin{array}{l}12 \\
(14.0 \%)\end{array}$ & $6(7 \%)$ & \\
\hline & $40-59$ & $104(81.3 \%)$ & $\begin{array}{l}10 \\
(7.8 \%)\end{array}$ & $14(10.9 \%)$ & \\
\hline & $>60$ & 20 (83.3\%) & $\begin{array}{l}4 \\
(16.7 \%)\end{array}$ & $0(0 \%)$ & \\
\hline \multirow[t]{2}{*}{ Sex } & Men & $66(74.2 \%)$ & $\begin{array}{l}15 \\
(16.9 \%)\end{array}$ & $8(9 \%)$ & \multirow[t]{2}{*}{.113} \\
\hline & Women & 191 (78.6\%) & $\begin{array}{l}22 \\
(9.1 \%)\end{array}$ & $30(12.3 \%)$ & \\
\hline \multirow[t]{2}{*}{ Nationality } & Saudi & $235(76.5 \%)$ & $\begin{array}{l}35 \\
(11.4 \%)\end{array}$ & 37 (12.1\%) & \multirow[t]{2}{*}{.379} \\
\hline & Non-Saudi & $22(88 \%)$ & $2(8 \%)$ & $1(4 \%)$ & \\
\hline \multirow[t]{4}{*}{ Educational level } & Elementary & $0(0 \%)$ & $0(0 \%)$ & $2(100 \%)$ & \multirow[t]{4}{*}{.001} \\
\hline & Secondary & 27 (73.0\%) & $\begin{array}{l}7 \\
(18.9 \%)\end{array}$ & $3(8.1 \%)$ & \\
\hline & University & $178(75.7 \%)$ & $\begin{array}{l}27 \\
(11.5 \%)\end{array}$ & $30(12.8 \%)$ & \\
\hline & $\begin{array}{l}\text { Higher } \\
\text { education }\end{array}$ & $52(89.7 \%)$ & $\begin{array}{l}3 \\
(5.2 \%)\end{array}$ & $3(5.2 \%)$ & \\
\hline \multirow[t]{2}{*}{$\begin{array}{l}\text { How many times did you } \\
\text { use the service? }\end{array}$} & Once & $177(76.3 \%)$ & $\begin{array}{l}32 \\
(13.8 \%)\end{array}$ & $23(9.9 \%)$ & \multirow[t]{2}{*}{0.038} \\
\hline & More than once & $80(80 \%)$ & $5(5 \%)$ & $15(15.0 \%)$ & \\
\hline \multirow[t]{4}{*}{$\begin{array}{l}\text { For what reason did you } \\
\text { use the service? }\end{array}$} & New complaint & $64(74.4 \%)$ & $\begin{array}{l}18 \\
(20.9 \%)\end{array}$ & $4(4.7 \%)$ & \multirow[t]{4}{*}{.001} \\
\hline & Follow-up & 59 (73.8\%) & $\begin{array}{l}3 \\
(3.8 \%)\end{array}$ & $18(22.5 \%)$ & \\
\hline & Emergency & 49 (81.7\%) & $\begin{array}{l}4 \\
(6.7 \%)\end{array}$ & $7(11.7 \%)$ & \\
\hline & $\begin{array}{l}\text { Refill } \\
\text { medication }\end{array}$ & 38 (82.6\%) & $\begin{array}{l}3 \\
(6.5 \%)\end{array}$ & $5(10.9 \%)$ & \\
\hline
\end{tabular}




\begin{tabular}{|c|c|c|c|c|c|}
\hline \multicolumn{2}{|l|}{ Characteristics } & \multirow{2}{*}{$\begin{array}{l}\text { Strongly } \\
\text { agree/agree }\end{array}$} & \multirow{2}{*}{$\begin{array}{l}\begin{array}{l}\text { Not } \\
\text { sure }\end{array} \\
9(15 \%)\end{array}$} & \multirow{2}{*}{$\begin{array}{l}\begin{array}{l}\text { Strongly } \\
\text { disagree/disagree }\end{array} \\
4(6.7 \%)\end{array}$} & \multirow[t]{2}{*}{$\begin{array}{l}P \\
\text { value }\end{array}$} \\
\hline & $\begin{array}{l}\text { COVID-19 } \\
\text { related }\end{array}$ & & & & \\
\hline \multirow[t]{5}{*}{$\begin{array}{l}\text { What was the outcome } \\
\text { or treatment from your } \\
\text { call consultation? }\end{array}$} & $\begin{array}{l}\text { Reassurance } \\
\text { and self- } \\
\text { management }\end{array}$ & 97 (82.9\%) & $\begin{array}{l}13 \\
(11.1 \%)\end{array}$ & $7(6 \%)$ & \multirow[t]{5}{*}{.02} \\
\hline & $\begin{array}{l}\text { Prescribed } \\
\text { drugs }\end{array}$ & $104(80 \%)$ & $\begin{array}{l}14 \\
(10.8 \%)\end{array}$ & $12(9.2 \%)$ & \\
\hline & Appointment & $13(76.5 \%)$ & $\begin{array}{l}2 \\
(11.8 \%)\end{array}$ & $2(11.8 \%)$ & \\
\hline & $\begin{array}{l}\text { Ambulance } \\
\text { sent }\end{array}$ & $9(75 \%)$ & $\begin{array}{l}1 \\
(8.3 \%)\end{array}$ & $2(16.7 \%)$ & \\
\hline & Other & $34(60.7 \%)$ & $\begin{array}{l}7 \\
(12.5 \%)\end{array}$ & $15(26.8 \%)$ & \\
\hline
\end{tabular}

As can be observed, there was a significant association with age $(P=.04)$ and with using the service once or multiple times $(P=.03)$. The association with the outcome of the consultation was even more significant $(P=.02)$ than that for age or the number of times using the service. The strongest association in terms of using the service again was the reasons for using the service $(P=.01)$ and educational level $(P=.01)$.

\subsection{Qualitative analysis of the main problems that the participants encountered during the teleconsultation experience}

At the end of the questionnaire, the participants were asked an open-ended question about any problems they encountered during their teleconsultation. After ruling out the positive and unrelated comments, only $41(10 \%)$ negative comments were included for further analysis (Table 7). A significant majority (70\%) of the complaints with the service were related to the long waiting time for a consultation. 
Table 7

Main problems encountered the participants during the teleconsultation.

\begin{tabular}{|lll|}
\hline Theme & Comments & $\mathbf{N}(\%)$ \\
\hline $\begin{array}{l}\text { Response and waiting } \\
\text { time }\end{array}$ & Delayed response. & (70\%) \\
\cline { 2 - 3 } & $\begin{array}{l}\text { Long waiting time. } \\
\text { Unsolved medical issue }\end{array}$ & $\begin{array}{l}\text { The doctor did not understand my medical issues. } \\
\text { No response. }\end{array}$ \\
\hline $\begin{array}{l}\text { They gave me an appointment without explaining my } \\
\text { problem. }\end{array}$ & \\
\hline My problem was not solved. & $3(\%)$ \\
\hline Technical issues & The doctor's voice was not clear. & \\
\hline
\end{tabular}

\section{Discussion}

This study aimed to evaluate the patients' perceptions and satisfaction regarding teleconsultations during the COVID-19 pandemic in Jeddah, Saudi Arabia. Factors that could influence patient satisfaction with the service were also considered. To our knowledge, this is the first study to assess patient satisfaction regarding teleconsultation in Saudi Arabia during the COVID-19 pandemic.

The demographic data indicated that the majority of the participants in this study were women between the ages of 30 and 59 with a college degree. It is worth mentioning that the results of this study showed that $70 \%$ of the study population used teleconsultation only once. This could be explained, to some extent, by the reported outcomes from their calls. The outcomes of the majority of the teleconsultation services result in either the patients' reassurance or medication prescription to them. However, more studies are needed to explore this area in-depth.

Although several studies $[16,17]$ have indicated that these characteristics generally correlate with lower patient satisfaction, we found that patient satisfaction with the teleconsultation services was high across all domains tested. In over $70 \%$ of the cases, the participants felt that the doctor provided them with an appropriate management plan, that they understood their condition better, and that they were able to receive and convey information over the phone.

Similarly, in terms of satisfaction with the experience, $>80 \%$ of the participants were satisfied with the fact that they did not need a physical meeting with the doctor. As first reported by Newbould et al.,[18] patient dissatisfaction with teleconsultation seems to increase when the patient still needs to have a physical visit despite the teleconsultation. Hence, it can be argued that the high rates of satisfaction, in this case, were obtained in part because the vast majority of the participants did not need to visit the doctor's office following the teleconsultation. 
Several demographic variables appeared to impact satisfaction levels. In some cases, these elements were found to concur with previous reports. $[16,17,18]$ Such aspects include older individuals and their likelihood of being satisfied with the service. Newbould et al.[18] stated that younger people are quicker to find solutions to their medical issues and therefore tend to be more dissatisfied with the services provided than older individuals. Similarly, the outcome of the service was also found to influence patient satisfaction regardless of the nature of the outcome. Some divergent aspects were also noted. Contrary to previous findings, $[18,19,20]$ this study found that patients with lower levels of education tended to be more dissatisfied with the service.

Finally, contrary to other reports, sex was not found to influence levels of satisfaction.

Hence, by corroborating the results obtained by this study with similar studies, it appears that patient satisfaction with teleconsultation services is largely dictated by the reasons for which the service is requested, the outcomes of the consultation, and by specific patient characteristics.

Despite experiencing higher levels of dissatisfaction after using the service more than once, participants in this study were also more likely to use the service again if they had used it before. The interpretation of these results could be limited by the circumstances created by the COVID-19 pandemic.[21] Repeated use of the service might occur for these patients, if they lack other alternatives. Those with higher educational levels were also more likely to use the service and experienced greater satisfaction with the service, contrary to other findings. Finally, the reasons for use and the outcomes of the service also had a significant impact on the likelihood of reuse.

The reasons for service dissatisfaction identified by this study have also been reported elsewhere.[18] Over $70 \%$ of participants were dissatisfied with the long waiting time for a consultation, and $9 \%$ were unhappy because, despite the consultation, they had to attend a physical appointment. Only $3 \%$ had experienced a technical difficulty, which might indicate that the quality of the service could be influenced by the infrastructure available to carry out the teleconsultation.

Despite showing an overall high satisfaction rate with the teleconsultation service, these results must be considered with caution. First, the patient sample presents various homogenous characteristics, especially in terms of sex and education. These aspects limit the generalizability of the study across Saudi Arabia. It is therefore recommended that future studies include more diverse participant samples when assessing patient satisfaction with teleconsultations. Response bias is another limitation that can affect the outcome. Furthermore, the results of this study cannot be generalized to all medical subspecialties. The applicability of teleconsultation in various subspecialties must be assessed individually. Also, assessing the physicians' perceptions toward teleconsultation was beyond the scope of this study. It is worth mentioning that the adoption of telemedicine by health professionals is limited in Saudi Arabia despite the efforts and support provided by the Saudi MOH.[2, 6, 22] A recent systemic review highlighted the factors that influenced the adoption of telemedicine in Saudi Arabia, which included a lack of healthcare provider knowledge and limited publications in support of such services.[5] 
Thus, it is crucial to increase the knowledge of healthcare providers about teleconsultation services and to incorporate this topic in all training programs and university medical curricula.

\section{Conclusions}

We conclude that patients were generally satisfied with the teleconsultation service implemented in Jeddah during the COVID-19 pandemic. More studies will be necessary to obtain an in-depth understanding of patients' perception and satisfaction with these services as well as the necessary logistics. However, the telephone service could be extended to other medical areas and appears to function well as a viable alternative to physical consultations. Given the current circumstances created by the COVID-19 pandemic, investigating these services in terms of patient satisfaction, service quality, and potential improvements is a global necessity. The results of this study could shape the future of practicing medicine in Saudi Arabia and will help with establishing guidelines for conducting teleconsultation services. However, more studies are required to provide evidence-based data for teleconsultation in various medical subspecialties and specific patient populations.

\section{List Of Abbreviations}

$\mathrm{MOH}$ Ministry of Health

SD Standard deviation

SR Swain-Eng RJ

\section{Declarations}

\section{Ethics approval and consent to participate}

This study was approved by the Unit of Biomedical Ethics Research Committee at the Faculty of Medicine, King Abdulaziz University (Reference No. 229-20), dated May 4, 2020.

\section{Consent for publication}

Not applicable.

\section{Availability of data and materials}

Data that support the findings in the current study are available from the corresponding author on reasonable request.

\section{Competing interests}

The authors declare that they have no competing interests. 


\section{Funding}

This study did not receive any specific grant from funding agencies in the public, commercial, or not-forprofit sectors.

\section{Authors' contributions}

Idea development and study design: MM acquired and analyzed data, and drafting the first manuscript. RM acquired and analyzed data, and submitted of the manuscript. FK acquired and analyzed data, and critical revision of paper. All authors read and approved the final manuscript.

\section{Acknowledgements}

The authors would like to thank all participants who completed the questionnaire during the COVID-19 pandemic. In addition, the authors would like to acknowledge Enago (www.enago.com) for their English language editing service.

\section{References}

1. WHO. A health telematics policy in support of WHO's health for all strategy for global health development: report of the WHO group consultation on health telematics, 11-16 December, Geneva 1997. Geneva: World Health Organisation; 1998. Accessed 23 July 2020.

2. El-Mahalli AA, El-Khafif SH, Al-Qahtani MF. Successes and challenges in the implementation and application of telemedicine in the eastern province of Saudi Arabia. Perspect Health Inf Manag. 2012;9:1-27.

3. Downes MJ, Mervin MC, Byrnes JM, Scuffham PA. Telephone consultations for general practice: a systematic review. Syst Rev. 2017;6(1):128.

4. Wechsler LR, Tsao JW, Levine SR, Swain-Eng RJ, Adams RJ, Demaerschalk BM, Hess DC, Moro E, Schwamm LH, Steffensen S, et al. Teleneurology applications: Report of the Telemedicine Work Group of the American Academy of Neurology. Neurology. 2013;80(7):670-6.

5. Alshahrani A, Stewart D, MacLure K. A systematic review of the adoption and acceptance of eHealth in Saudi Arabia: Views of multiple stakeholders. Int J Med Inform. 2019;128:7-17.

6. Ullah S, Maghazil AM, Qureshi AZ, Tantawy S, Moukais IS, Aldajani AA. Knowledge and attitudes of rehabilitation professional toward telerehabilitation in Saudi Arabia: A cross-sectional survey. Telemed J E Health. 2020. doi:10.1089/tmj.2020.0016.

7. COVID 19 Dashboard. Saudi Arabia [https://covid19.moh.gov.sa] Accessed 23 July 2020.

8. Portnoy J, Waller M, Elliott T. Telemedicine in the era of COVID-19. J Allergy Clin Immunol Pract. 2020;8(5):1489-91.

9. Calton B, Abedini N, Fratkin M. Telemedicine in the time of coronavirus. J Pain Symptom Manage. 2020;60(1):e13-4. 
10. Latifi R, Doarn CR. Perspective on COVID-19: finally, telemedicine at center stage. Telemed J E Health. 2020. doi:10.1089/tmj.2020.0132.

11. Liu S, Yang L, Zhang C, Xiang YT, Liu Z, Hu S, Zhang B. Online mental health services in China during the COVID-19 outbreak. Lancet Psychiatry. 2020;7(4):e17-8.

12. Vidal-Alaball J, Acosta-Roja R, Pastor Hernandez N, Sanchez Luque U, Morrison D, Narejos Perez S, Perez-Llano J. Salvador Verges A, Lopez Segui F. Telemedicine in the face of the COVID-19 pandemic. Aten Primaria. 2020;52(6):418-22.

13. Capozzo R, Zoccolella S, Musio M, Barone R, Accogli M, Logroscino G. Telemedicine is a useful tool to deliver care to patients with Amyotrophic Lateral Sclerosis during COVID-19 pandemic: results from Southern Italy. Amyotroph Lateral Scler Frontotemporal Degener. 2020:1-7.

14. Layfield E, Triantafillou V, Prasad A, Deng J, Shanti RM, Newman JG, Rajasekaran K. Telemedicine for head and neck ambulatory visits during COVID-19: Evaluating usability and patient satisfaction. Head Neck. 2020;42(7):1681-89.

15. Kruse CS, Krowski N, Rodriguez B, Tran L, Vela J, Brooks M. Telehealth and patient satisfaction: a systematic review and narrative analysis. BMJ Open. 2017;7(8):e016242.

16. Woods SE, Heidari Z. The influence of gender on patient satisfaction. J Gend Specif Med. 2003;6(4):30-5.

17. Lopez C, Valenzuela JI, Calderon JE, Velasco AF, Fajardo R. A telephone survey of patient satisfaction with realtime telemedicine in a rural community in Colombia. J Telemed Telecare. 2011;17(2):83-7.

18. Newbould J, Ball S, Abel G, Barclay M, Brown T, Corbett J, Doble B, Elliott M, Exley J, Knack A, et al. A 'telephone first' approach to demand management in English general practice: a multimethod evaluation. In: A 'telephone first' approach to demand management in English general practice: a multimethod evaluation. edn. Southampton (UK); 2019.

19. Tabekhan AK, Alkhaldi YM, Alghamdi AK. Patients satisfaction with consultation at primary health care centers in Abha City, Saudi Arabia. J Family Med Prim Care. 2018;7(4):658-63.

20. Prakash B. Patient satisfaction. J Cutan Aesthet Surg. 2010;3(3):151-5.

21. Wosik J, Fudim M, Cameron B, Gellad ZF, Cho A, Phinney D, Curtis S, Roman M, Poon EG, Ferranti J, et al. Telehealth transformation: COVID-19 and the rise of virtual care. J Am Med Inform Assoc. 2020;27(6):957-62.

22. Albarrak Al, Mohammed R, Almarshoud N, Almujalli L, Aljaeed R, Altuwaijiri S, Albohairy T. Assessment of physician's knowledge, perception and willingness of telemedicine in Riyadh region, Saudi Arabia. J Infect Public Health 2019;S1876-0341(19)30139-X.. 